\title{
Convergence to Equilibrium of the Stochastic Heisenberg Model
}

\author{
William D. Wick \\ Mathematics Department, University of Washington, Seattle, WA 98195, USA
}

\begin{abstract}
The stochastic Heisenberg model is a probabilistic model of the time evolution of a classical Heisenberg ferromagnet. It is proved that the stochastic process converges to equilibrium at sufficiently high temperatures, and that the equilibrium state is a Gibbs state of the Hamiltonian possessing the global Markov property. The principal technique employed is the expansion of observables on the state space into Laplace series.
\end{abstract}

\section{Introduction}

The stochastic Heisenberg model was introduced by William Faris in [1] as a probabilistic model of the time evolution of a classical Heisenberg ferromagnet. He constructed an infinite-dimensional Markov diffusion process whose state space is a countable product of spheres, and which has two components. The first is a random motion, the product of Brownian motions on the individual spheres. The second is a deterministic drift generated by a vector field containing the interaction terms. The latter is formally the negative of the gradient of the Hamiltonian, multiplied by the inverse temperature.

In a second paper [2] the same author studied the equilibrium state of the model and proved that it is unique and analytic in the inverse temperature for sufficiently high temperatures. This was obtained by expanding the equilibrium state into a series of Rayleigh-Schrödinger type around a product measure, and proving convergence of the series in an appropriate norm.

The main theorem of the present paper (Theorem 1 of Sect. III) establishes convergence of this process with arbitrary initial state to the equilibrium state at high temperature, for a large class of models of the type studied by Faris. The principal technique we employ is the expansion of functions on the state space into series of eigenfunctions of the infinite-dimensional Laplacian (Laplace series.) We include an existence theorem for the process of a different sort than Faris's. We also give a discussion of the relationship between the equilibrium state of the stochastic model and the Gibbs state of the Hamiltonian and show how the global Markov property of the equilibrium state may be deduced from Faris's uniqueness theorem. 
We now define the class of models we are interested in. Let $M$ be a compact, homogeneous Riemannian manifold with metric tensor $g$. $M$ will play the role of 'spin space' for an individual 'spin'. Let $S$ be a countable set of 'sites' equipped with a metric $d($,) (we assume also that $x \in S$ and $r<\infty$ imply that $\{y: d(y, x) \leqq r\}$ is finite.) The state space will be $\Xi=M^{S}=\prod_{x \in S} M_{x}$, with $M_{x}=M$ the manifold at site $x \in S$. We write $\xi \in \Xi$ and $\xi(x) \in M_{x}$ for the 'spin' at site $x \in S$. Let $\Delta=\sum_{x \in S} \Delta_{x}$ be the (infinite-dimensional) Laplacian; $\Delta_{x}$ is defined to be $\nabla_{x} \cdot g^{-1} \cdot d_{x}$, with $\nabla_{x}$ the covariant derivative and $d_{x}$ the differential with respect to $\xi(x) \in M_{x} . \Delta$ is well-defined on smooth functions on $\Xi$ of finitely many manifolds.

Let $X$ be a vector field on $\Xi$ and $X \cdot d$ the corresponding first-order differential operator. $X \cdot d$ is defined on the same domain as $\Delta$ by: $X \cdot d=\sum_{x \in S} X_{x} \cdot d_{x}$, where $X_{x}$ is a function of $\xi \in \Xi$ whose value is a tangent vector to $M_{x}$ at $\xi(x)$. We assume that $X$ satisfies the following 'finite-range' and smoothness condition: $X_{x}$ depends only on the variables $\xi(y)$ with $d(y, x) \leqq r(x)<\infty$ and is a $C^{\infty}$ function of these variables. In the models studied in [1] and the present paper, $X$ is formally a gradient: $X=-\nabla H$, where $H$ (the Hamiltonian) is a scalar function on $\Xi$. Typically $H$ is given by a divergent sum of smooth 'potential' functions each of which depends on only finitely many manifolds and we may define $X$ rigorously by setting $X_{x}=-\nabla_{x} H=-g^{-1} d_{x} H$. Then if $H$ contains only finite-range interactions $X$ will satisfy the finite-range condition.

The generator of our Markov process will be constructed by setting

$$
\Omega=\Delta+T^{-1} X \cdot d
$$

on the domain indicated above; $T$ is a positive constant proportional to the temperature. With some additional hypotheses, Faris in [1] proved via the HilleYosida Theorem that the closure of $\Omega$ is the generator of a Markov semigroup $\{S(t): t \geqq 0\}$ on $C(\Xi)$, the Banach space of continuous functions on $\Xi$ (a compact Hausdorf space), with the uniform norm $\|f\|_{u}=\sup _{\xi \in \Xi}|f(\xi)|$. (The $S(t)$ form a strongly continuous semigroup of positivity preserving linear contractions on $C(\Xi)$ leaving the function 1 invariant. For the definition of Markov semigroup and a discussion of the Hille-Yosida theorem in this context see [1], [3].) From the semigroup operators one can construct transition functions and a strong Markov diffusion process in the usual way [4].

We will call a probability measure on $\Xi$ a state. An invariant state $\mu$ for $\{S(t)\}$, i.e. one for which

$$
\int d \mu S(t) f=\int d \mu f
$$

for all $f \in C(\Xi)$ and $t \geqq 0$, will be called an equilibrium state. We will say that the process converges to equilibrium if $\pi S(t) \rightarrow \mu$ (in the weak* topology on measures) as $t \rightarrow \infty$ for an arbitrary initial state $\pi$ on $\Xi$. ( $\mu$ is then necessarily unique.) We avoid the term 'ergodicity' in this context since the time-shift operator may be ergodic on the path space of the process even if the equilibrium state is non-unique. See [5].

Our main theorem (which gives some conditions on the generator implying 
convergence to equilibrium of the corresponding process) was inspired by the proof of Liggett in [3] of a theorem of Holley and Stroock [6]. Their theorem gave existence and convergence to equilibrium of stochastic Ising models whose spinflip rate functions have absolutely convergent Fourier expansions. Our proof follows that of Liggett but is complicated by the necessity of using the more general Laplace series.

The plan of the paper is as follows. In Sect.II we introduce an appropriate space of functions with absolutely convergent Laplace series. In Sect.III the main theorem is proved, and in Sect.IV we compute the temperature ranges for which this theorem yields convergence to equilibrium, for two important special cases: the planerotor and classical Heisenberg models. These correspond to taking $S=Z^{v}, v \geqq 1 ; M=S^{n}$, the unit sphere in $R^{n+1}$, for $n=1$ and 2 (respectively); and

$$
H=-\frac{1}{2} \sum_{\substack{x, y \in S \\|x-y|=1}} \xi(x) \cdot \xi(y)
$$

(the '.' denotes dot product in $R^{n+1}$ ). In Sect.V we discuss the relationship between Gibbs states of the Hamiltonian and equilibrium states of the stochastic process for the general model with $X=-\nabla H$ (formally). We also derive the global Markov property of the equilibrium state from Faris's uniqueness theorem. Finally in an appendix we prove a different (and stronger) version of Theorem 1 for the planerotor models (i.e. models with $M=S^{1}$.) As a corollary, we obtain convergence to equilibrium for these models over a wider range of temperatures.

\section{Functions with Absolutely Convergent Laplace Series}

In this section we define a space of functions on $\Xi$ which have absolutely convergent expansions in eigenfunctions of the Laplacian (Laplace series). We begin by constructing the appropriate space of functions on $M$ and then extend the construction to product spaces.

Let $M$ be a compact and orientable $C^{\infty}$ Riemannian manifold. Let $g$ denote the metric tensor, $\Delta$ the Laplacian (Laplace-Beltrami operator), and $\sigma$ the volume measure on $M$ (normalized so that $\sigma(M)=1$ ). We assume in addition that $M$ is homogeneous: There exists a Lie group $G$ acting transitively on $M$ leaving $g$ invariant. We shall need the following well-known facts concerning the Laplacian as an operator on $L^{2}(M, \sigma)$ with domain the $C^{2}$ functions on $M$ :

(i) $\Delta$ is symmetric and has purely discrete spectrum lying in $(-\infty, 0]$. Let $0=\lambda(0)>\lambda(1)>\lambda(2)>\ldots$ be the eigenvalues of $\Delta$ with corresponding eigenspaces $H_{l}, l=0,1,2, \ldots$.

(ii) each $H_{l}$ is finite-dimensional and consists of $C^{\infty}$ functions.

(iii) The action of $\mathrm{G}$ on functions on $M$ induces a unitary representation of $G$ in each $\mathrm{H}_{l}$.

For each $l \geqq 0$ let $\left\{Y_{l, m}\right\}$ be an orthonormal basis of $H_{l}$ ( $m$ runs over some index set of cardinality equal to $\operatorname{dim} H_{l}$ ). Then we may write the orthogonal projection 
$P_{l}$ onto $H_{l}$ as an integral operator with kernel

Thus if $f \in L^{2}(M, \sigma)$,

$$
K_{l}(\xi, \eta)=\sum_{m} Y_{l, m}(\xi) \bar{Y}_{l, m}(\eta)
$$

$$
P_{l} f(\xi)=\int_{M} K_{l}(\xi, \eta) f(\eta) d \sigma(\eta) .
$$

Since $P_{l}$ is a projection we also have

$$
P_{l} f(\xi)=\int_{M} K_{l}(\xi, \eta) P_{l} f(\eta) d \sigma(\eta) .
$$

Applying Schwarz's inequality gives

$$
\begin{aligned}
\left|P_{l} f(\xi)\right| & \leqq\left(\int\left|K_{l}(\xi, \eta)\right|^{2} d \sigma(\eta)\right)^{1 / 2}\left(\int\left|P_{l} f(\zeta)\right|^{2} d \sigma(\zeta)\right)^{1 / 2} \\
& =K_{l}(\xi, \xi)^{1 / 2}\left\|P_{l} f\right\|_{2} .
\end{aligned}
$$

( \|\|$_{2}$ is the $L^{2}$-Norm.) Using (iii) it is easily seen that $K_{l}(\xi, \xi)$ is a constant equal to $\operatorname{tr} P_{l}=\operatorname{dim} H_{l}$.

Definition. $A(M) \equiv\left\{f \in L^{2}(M, \sigma): \sum_{l} w(l)\left\|P_{l} f\right\|_{2}<\infty\right.$, with $\left.w(l)=\left(\operatorname{dim} H_{l}\right)^{1 / 2}\right\}$.

From (1) we see that if $f \in A(M)$ then the Laplace series $\sum P_{l} f(\xi)$ converges absolutely and uniformly to $f(\xi)$. Thus $A(M) \subset C(M)$. $A(M)$ generalizes the notion of absolutely convergent Fourier series to arbitrary compact, homogeneous Riemannian spaces (equivalently, arbitrary compact Lie groups). However, there are other natural generalizations (see [7] for a discussion of this). In [8] Peetre proved that a sufficiently smooth function is in $A(M)$. In fact from the proof of Theorem One of [8], it follows that $f \in D^{n / 4,1} \Rightarrow f \in A(M)$, where $D^{n / 4,1}$ is a certain 'interpolation space' and $n=\operatorname{dim} M$. It is known that $D\left(\Delta^{N}\right) \subset D^{n / 4,1}$ if $N>n / 4$.

Of course the exemplar of this construction is for the sphere $S^{n}$, the unit sphere in $R^{n+1}$, with $\Delta$ the usual Laplacian (in the angular variables). We have $S O(n+1, R)$ acting transitively on $M, H_{l}$ is the space of surface spherical harmonics of degree $l$, and $\lambda(l)=-l(l+n-1)$. See e.g. [9].

We now generalize this construction to $\Xi=M^{S}$. Let now $\Delta=\sum_{x} \Delta_{x}$ be the Laplacian on $\Xi$ and $d \sigma(\xi)=\prod_{x} d \sigma(\xi(x))$ the volume measure. Define

$$
L=\left\{\mathbf{I} \mid \mathbf{l}: S \rightarrow Z^{+} \text {and } l(x)=0 \text { except for finitely many } x \in S\right\} .
$$

Then $L^{2}(\Xi, \sigma)$ admits a decomposition

$$
L^{2}(\Xi, \sigma)=\oplus \sum_{1} H_{1}
$$

where $H_{1}$ is the tensor product

$$
H_{1}=\otimes \prod_{x} H_{l(x)} .
$$

Each $H_{1}$ is an eigenspace of $\Delta$ of eigenvalue $\lambda(\mathbf{l})=\sum_{x} \lambda(l(x))$ and has dimension

$$
\operatorname{dim} H_{1}=\prod_{x} \operatorname{dim} H_{l(x)}
$$


We may construct an orthonormal basis of $H_{1}$ by setting

$$
Y_{1, \mathbf{m}}(\xi)=\prod_{x} Y_{l(x), m(x)}(\xi(x))
$$

Letting $P_{1}$ denote the orthogonal projection onto $H_{1}$ we find as before that $P_{1}$ is an integral operator with kernel

$$
K_{\mathbf{l}}(\xi, \eta)=\sum_{\mathbf{m}} Y_{\mathbf{1}, \mathbf{m}}(\xi) \bar{Y}_{\mathbf{1}, \mathbf{m}}(\eta)
$$

and a calculation identical to that preceding (1) gives, for $f \in L^{2}(\Xi, \sigma)$,

$$
\left|P_{1} f(\xi)\right| \leqq K_{1}(\xi, \xi)^{1 / 2}\left\|P_{1} f\right\|_{2},
$$

with

$$
\begin{aligned}
K_{\mathbf{l}}(\xi, \xi) & =\prod_{x} K_{l(x)}(\xi(x), \xi(x)) \\
& =\operatorname{dim} H_{\mathbf{r}}
\end{aligned}
$$

Definition $A(\Xi) \equiv\left\{f \in L^{2}(\Xi, \sigma): \sum_{\mathbf{l} \in L} w(\mathbf{l})\left\|P_{\mathbf{l}} f\right\|_{2}<\infty\right.$, with $\left.w(\mathbf{l})=\left(\operatorname{dim} H_{1}\right)^{1 / 2}\right\} . A(\Xi)$ is a Banach space with norm

$$
\|f\|_{a}=\sum_{\mathbf{l}} w(\mathbf{l})\left\|P_{1} f\right\|_{2}
$$

Note that $\|f\|_{u} \leqq\|f\|_{a}$ for $f \in A(\Xi)$.

Applying Theorem One of [8] to the manifold $M^{\Lambda}$ and Laplacian $\Delta_{\Lambda}=\sum_{x \in A} \Delta_{x}$ with $\Lambda \subset S$ finite, we see that $C^{\infty}$ functions of finitely many manifolds are in $A(\Xi)$. [Note that on $M^{\Lambda}$, Peetre's theorem gives convergence of the series

$$
\sum_{\lambda} w(\lambda)\left\|P_{\lambda} f\right\|_{2}
$$

for sufficiently smooth $f$, where the sum is over the eigenvalues $\lambda$ of $\Delta_{A}$ and $P_{\lambda}$ projects on the corresponding eigenspace $H_{\lambda}$, with $w(\lambda)=\left(\operatorname{dim} H_{\lambda}\right)^{1 / 2}$. However by Cauchy's inequality,

$$
\begin{aligned}
\sum_{\mathbf{l}: \lambda(\mathbf{l})=\lambda} w(\mathbf{l})\left\|P_{\mathbf{l}} f\right\|_{2} & \leqq\left(\sum_{\mathbf{l}: \lambda(\mathbf{l})=\lambda} w(\mathbf{l})^{2}\right)^{1 / 2}\left(\sum_{\mathbf{l}: \lambda(\mathbf{l})=\lambda}\left\|P_{\mathbf{l}} f\right\|_{2}^{2}\right)^{1 / 2} \\
& =w(\lambda)\left\|P_{\lambda} f\right\|_{2},
\end{aligned}
$$

so that convergence of Peetre's series implies convergence of our series.] We conclude that $A(\Xi)$ is dense in $C(\Xi)$.

The following proposition will prove useful in the sequel. The proof (which is straightforward) is omitted.

Proposition 1. Let $H$ be a Hilbert space decomposed as: $H=\oplus \sum_{l \in L} H_{l}$, where $L$ is a countable index set. Let $w(l) \geqq 1$ be a function on L. Define the subspace $A=A\left(H,\left(H_{l}\right), w\right)$ of $H$ by: $f \in A$ iff the norm $\|f\|_{a} \equiv \sum_{l} w(l)\left\|f_{l}\right\|_{2}<\infty$, where $f$ has Fourier series $\sum_{l} f_{l}$ with $f_{l} \in H_{l}$. Then: 
(i) The dual $A^{*}$ of $A$ may be identified with the space of sequences $g=\left(g_{l}\right)_{l \in L}$ with $g_{l} \in H_{l}$, norm $\|g\|_{a}^{*}=\sup \left\|g_{l}\right\|_{2}$, and pairing

$$
\langle g, f\rangle=\sum_{l} w(l)\left(g_{l}, f_{l}\right)_{2} .
$$

(ii) Let $\Gamma_{l, l^{\prime}} \in B\left(H_{l^{\prime}}, H_{l}\right)$ be a matrix of bounded linear operators satisfying

$$
\sup _{l} w(l)^{-1} \sum_{l^{\prime}} w\left(l^{\prime}\right)\left\|\Gamma_{l^{\prime}, l}\right\|_{o p}<\infty
$$

(where $\|\cdot\|_{o p}$ denotes operator norm). Then the formula

$$
(\Gamma f)_{l}=\sum_{l^{\prime}} \Gamma_{l, l^{\prime}} f_{l^{\prime}}
$$

defines a bounded linear operator on $A$ with norm less than the number in (5).

Remark. If in the proposition we have $\operatorname{dim} H_{l}=1$ for all $l$, then the converse of part (ii) holds: Every bounded linear operator on $A$ is given as in (6) by a matrix with the number in (5) finite. This remains true if the dimensions are bounded by a finite constant but is false in the unbounded case. (The author thanks D. Ragozin for this remark.)

\section{Existence of the Process and Convergence to Equilibrium}

In order to state simply the main theorem we introduce some additional notation. Set $A_{x}=\Delta_{x}$ and $B_{x}=T^{-1} X_{x} d_{x}$ for $x \in S$. Then $A=\sum_{x} A_{x}, B=\sum_{x} B_{x}$ and $\Omega=A+B$. Let $\Lambda_{n}, n=1,2, \ldots$, be an increasing sequence of finite subsets of $S$ with union $S$ and let $B_{n}=\sum_{x \in A_{n}} B_{x}$ and $\Omega_{n}=A+B_{n}$. We shall need also the inverse operators $A^{-1}$ and $A_{x}^{-1}$ for $x \in S$ which we define on $\oplus \sum_{1 \neq 0} H_{1}$ (respectively on $\oplus \sum_{1: l(x) \neq 0} H_{l}$ ) so that $A A^{-1}=I$ (respectively $A_{x} A_{x}^{-1}=I$ ) and set equal to zero on the orthogonal complements of these spaces. Finally if $\Gamma$ is a linear operator on $L^{2}(\Xi, \sigma)$ define

$$
\|\Gamma \mid\|=\sup _{\mathbf{l}} w(\mathbf{l})^{-1} \sum_{\mathbf{l}^{\prime}} w\left(\mathbf{l}^{\prime}\right)\left\|P_{\mathbf{l}^{\prime}} \Gamma P_{\mathbf{1}}\right\|_{o p} .
$$

In the theorem below all operators are assumed to have as domain the $C^{2}$ functions of finitely many manifolds, and a bar over an operator denotes closure of the operator. The $\bar{\Omega}_{n}$ are generators since they are sums of finite-dimensional generators [1].

Theorem 1. Assume that there exists $\alpha<1$ such that

$$
\| B_{x} \circ A_{x}^{-1}|| \leqq \alpha
$$

for all $x \in S$. Then:

(i) $\bar{\Omega}$ generates a Markov semigroup on $C(\Xi) . \bar{\Omega}_{n} \rightarrow \bar{\Omega}$ in the sense of strong resolvent convergence: For all $\kappa>0$ and $f \in C(\Xi)$,

$$
\left(I-\kappa \bar{\Omega}_{n}\right)^{-1} f \rightarrow(I-\kappa \bar{\Omega})^{-1} f
$$

in uniform norm. 
(ii) The semigroup $\{S(t): t \geqq 0\}$ generated by $\bar{\Omega}$ has a unique invariant state $\mu$ and for any $g \in A(\Xi)$,

$$
\left\|S(t) g-\int g d \mu\right\|_{u} \leqq 2\|g\|_{a} \exp \left(-t \lambda_{1}(1-\alpha)\right)
$$

where $\lambda_{1}=\inf _{\substack{l \neq 0 \\ \text { f }}}(-\lambda(l))$. Thus if $\pi$ is any initial state on $\Xi$,

$$
\pi S(t) \rightarrow \mu
$$

in the weak* topology on measures.

Proof. The line of argument follows that in [3]; for the existence proof see also the lemma in Sect. 3 of $[1]$. $(A(\Xi)$ plays the role of Faris's auxiliary Banach space $W$ ). We begin by proving two lemmas which provide the necessary a priori estimates.

Lemma 1. Condition (2) implies the uniform bound (for all $\mathbf{l}, \mathbf{l}^{\prime}$ with $\mathbf{l}^{\prime} \neq 0$ ):

$$
\left\|P_{1} B_{n} \circ A^{-1} P_{1^{\prime}}\right\|_{o p} \leqq \Gamma_{1,1^{\prime}}
$$

where $\Gamma_{1, \mathbf{1}^{\prime}}$ is a matrix with positive entries (independent of $n$ ) such that

Proof.

$$
\sup _{\mathbf{l}^{\prime}} w\left(\mathbf{l}^{\prime}\right)^{-1} \sum_{\mathbf{l}} w(\mathbf{l}) \Gamma_{\mathbf{l}, \mathbf{l}^{\prime}} \leqq \alpha
$$

$$
\begin{aligned}
\left\|P_{1} B_{n} \circ A^{-1} P_{\mathbf{l}^{\prime}}\right\|_{o p} & =\left|\lambda\left(\mathbf{l}^{\prime}\right)\right|^{-1} \sum_{\substack{x: \\
x \in \Lambda_{n}, l^{\prime}(x) \neq 0}}\left\|P_{\mathbf{l}} B_{x} P_{\mathbf{l}^{\prime}}\right\|_{o p} \\
& \leqq\left|\lambda\left(\mathbf{l}^{\prime}\right)\right|^{-1} \sum_{\substack{x: \\
x \in \Lambda_{n}, \mathbf{l}^{\prime}(x) \neq 0}}\left|\lambda\left(\mathbf{l}^{\prime}(x)\right)\right|\left\|P_{1} B_{x} \circ A_{x}^{-1} P_{\mathbf{l}^{\prime}}\right\|_{o p}
\end{aligned}
$$

To obtain the bound (5) we drop the restriction $x \in \Lambda_{n}$ from the sum over $x$ on the right side of (7), and define $\Gamma_{1,1^{\prime}}$ as the resulting upper bound. We then obtain from (2),

$$
w\left(\mathbf{l}^{\prime}\right)^{-1} \sum_{\mathbf{l}} w(\mathbf{l}) \Gamma_{\mathbf{1}, \mathbf{l}^{\prime}} \leqq \alpha\left|\lambda\left(\mathbf{l}^{\prime}\right)\right|^{-1} \sum_{x}\left|\lambda\left(\mathbf{l}^{\prime}(x)\right)\right|=\alpha .
$$

In the next lemma let $D$ be the space of $C^{\infty}$ functions of finitely many manifolds. Lemma 2. Let $\kappa>0, f \in D$ and $\left(I-\kappa \Omega_{n}\right) f=g$. Then for each $\mathbf{l}$,

$$
\left\|P_{1} f\right\|_{2}(1+\kappa|\lambda(\mathbf{l})|) \leqq\left\|P_{1} g\right\|_{2}+\kappa \sum_{\mathbf{I}^{\prime} \neq 0}\left|\lambda\left(\mathbf{I}^{\prime}\right)\right| \Gamma_{\mathbf{1}, \mathbf{I}^{\prime}}\left\|P_{\mathbf{l}^{\prime}} f\right\|_{2}
$$

Proof. Since $f$ is $C^{\infty}$, both $f$ and $A f$ are in $A(\Xi)$. In particular the series $\Sigma \lambda(\mathbf{l}) P_{\mathbf{l}} f$ converges in $A(\Xi)$. This fact and the bounds in (5) and (6) (which imply that $B_{n}^{\circ} A^{-1}$ is a bounded operator on $A(\Xi)$ ) justify the following computation.

$$
\begin{aligned}
\sum\left(P_{\mathbf{l}} f\right)(1-\kappa \lambda(\mathbf{l})) & =\sum P_{\mathbf{l}} g+\kappa B_{n}\left(\sum P_{\mathbf{l}} f\right) \\
& =\sum P_{\mathbf{1}} g+\kappa \sum_{\mathbf{l} \neq 0} \lambda(\mathbf{l}) B_{n} \circ A^{-1} P_{\mathbf{l}} f .
\end{aligned}
$$

Projecting onto $H_{1}$ yields

$$
\left(P_{\mathbf{l}} f\right)(1-\kappa \lambda(\mathbf{l}))=P_{\mathbf{1}} g+\kappa \sum_{\mathbf{l}^{\prime} \neq 0} \lambda\left(\mathbf{I}^{\prime}\right) P_{\mathbf{l}^{\prime}} B_{n} \circ A^{-1} P_{\mathbf{1}^{\prime}} f,
$$

and the estimate (8) follows by taking $\|\cdot\|_{2}$ of both sides and using lemma one. 
We now begin the proof of (i). As in [1], $\Omega$ is a densely defined, dissipative operator and so has a closure $\bar{\Omega}$ with similar properties. The difficult part in verifying the hypotheses of the Hille-Yosida theorem is showing that the resolvents $(I-\kappa \bar{\Omega})^{-1}$, for $\kappa>0$, exist. For this we must show that $I-\kappa \bar{\Omega}$ has dense range (the range is closed since $\bar{\Omega}$ is dissipative). The semigroup operators are then constructed from the formula:

$$
S(t) f=\lim _{n \rightarrow \infty}(I-(t / n) \bar{\Omega})^{-n} f
$$

(the limit is in the uniform norm).

Let $g \in D$ and suppose that $g$ depends only on the manifolds $M_{x}$ with $x \in \Lambda$. Using the finite-range condition and existence and regularity theorems for elliptic P.D.E's we can solve $\left(I-\kappa \Omega_{n}\right) f_{n}=g$ for $f_{n} \in D$, if $n$ is large enough (such that $\left.\Lambda_{n} \supset \Lambda\right)$. [To do so it is necessary to check that the adjoint equation $\left(I-\kappa \Omega_{n}^{t}\right) f=0$, where $\Omega_{n}^{t}$ is the formal adjoint of $\Omega_{n}$ with respect to $\sigma$ and the equation is for a distribution $f$ on a finite-dimensional manifold, has only the solution $f \equiv 0$. For a proof of this see [1] or [10].] Lemma two applies and gives the estimates:

$$
\kappa|\lambda(\mathbf{I})|\left\|P_{1} f_{n}\right\|_{2} \leqq\left\|P_{1} g\right\|_{2}+\kappa \sum_{\mathbf{I}^{\prime}}\left|\lambda\left(\mathbf{l}^{\prime}\right)\right| \Gamma_{\mathbf{1}, \mathbf{I}^{\prime}}\left\|P_{\mathbf{1}^{\prime}} f_{n}\right\|_{2}
$$

The bound (6) translates into the bound $\|\Gamma\|_{o p}<1$ of the matrix $\Gamma$ operating on the weighted $l_{1}$-space obtained from $l_{2}(L)$ and the weight function $w$ as in Proposition 1. Hence $I-\Gamma$ is invertible on this space and we obtain from (10)

$$
u \leqq \kappa^{-1}(I-\Gamma)^{-1} v
$$

(componentwise), where

$$
u(\mathbf{l})=|\lambda(\mathbf{l})|\left\|P_{1} f_{n}\right\|_{2}
$$

and

$$
v(\mathbf{l})=\left\|P_{1} g\right\|_{2} .
$$

The conclusion is that for all $\mathbf{l}$,

$$
\kappa|\lambda(\mathbf{l})|\left\|P_{1} f_{n}\right\|_{2} \leqq r(\mathbf{l}),
$$

with $r(\mathbf{l}) \geqq 0$ independent of $n$ and

$$
\sum_{\mathbf{l}} w(\mathbf{l}) r(\mathbf{l})<\infty
$$

Now let $g_{n}=(I-\kappa \Omega) f_{n}$. We have

$$
\begin{aligned}
\left\|g-g_{n}\right\|_{u} & =\kappa\left\|\left(\Omega-\Omega_{n}\right) f_{n}\right\|_{u} \\
& \leqq \kappa \sum_{x \notin \Lambda_{n}}\left\|B_{x} f_{n}\right\|_{u} .
\end{aligned}
$$

Since $\|\cdot\|_{u}<\|\cdot\|_{a}$ the latter is

$$
\begin{aligned}
& \leqq \kappa \sum_{x \notin \Lambda_{n}}\left\|B_{x} f_{n}\right\|_{a} \\
& =\kappa \sum_{x \notin \Lambda_{n}}\left\|\left(B_{x}^{\circ} A_{x}^{-1}\right)\left(\sum_{1} \lambda(l(x)) P_{1} f_{n}\right)\right\|_{a}
\end{aligned}
$$




$$
\begin{aligned}
& \leqq \kappa \alpha \sum_{\substack { x \notin \Lambda_{n} \\
\begin{subarray}{c}{l(x) \neq 0 \\
l(x) \neq 0{ x \notin \Lambda _ { n } \\
\begin{subarray} { c } { l ( x ) \neq 0 \\
l ( x ) \neq 0 } }\end{subarray}} w(\mathbf{l})|\lambda(l(x))|\left\|P_{1} f_{n}\right\|_{2} \\
& \leqq \kappa \alpha \sum_{\substack{1: \\
\operatorname{supp} \mathbf{l} \Lambda_{n}^{c} \neq \phi}} w(\mathbf{l})|\lambda(\mathbf{l})|\left\|P_{1} f_{n}\right\|_{2} .
\end{aligned}
$$

(We have used (2) and part (ii) of Proposition 1 in the third inequality, and supp $\mathbf{I}=\{x: l(x) \neq 0\})$. From (11), (12) and dominated convergence we see that the last bound tends to zero as $n \rightarrow \infty$.

Thus $g_{n} \rightarrow g$ uniformly. Furthermore since $I-\kappa \Omega$ is dissipative,

$$
\left\|f_{n}-f_{m}\right\|_{u} \leqq\left\|g_{n}-g_{m}\right\|_{u} \rightarrow 0
$$

Thus $f_{n} \rightarrow f$ uniformly, $f \in D(\bar{\Omega})$ and $(I-\kappa \bar{\Omega}) f=g$. By the Hille-Yosida Theorem, $\bar{\Omega}$ generates a (Markov) semigroup on $C(\Xi)$. (3) follows from the lemma of [1].

We now prove (ii). Let $g \in D$ and $(I-\kappa \bar{\Omega}) f=g$. From the proof of (i), we obtain (8) holding for $f$ and $g$ since this inequality holds with $f_{n}$ in place of $f, P_{1} f_{n} \rightarrow P_{1} f$ uniformly for each 1 , and dominated convergence applies because of the bound (11). Multiplying both sides of $(8)$ by $w(\mathbf{l})$, summing over $\mathbf{l} \neq 0$ and using (6) we obtain

$$
\left(1+\kappa \lambda_{1}(1-\alpha)\right) \sum_{\mathbf{l} \neq 0} w(\mathbf{l})\left\|P_{1} f\right\|_{2} \leqq \sum_{\mathbf{l} \neq 0} w(\mathbf{l})\left\|P_{1} g\right\|_{2},
$$

where $\lambda_{1}=\inf _{l \neq 0}|\lambda(l)|$. We rewrite this as

$$
\sum_{\mathbf{l} \neq 0} w(\mathbf{l})\left\|P_{\mathbf{l}}(I-\kappa \bar{\Omega})^{-1} g\right\|_{2} \leqq\left(1+\kappa \lambda_{1}(1-\alpha)\right)^{-1} \sum_{\mathbf{l} \neq 0} w(\mathbf{l})\left\|P_{\mathbf{1}} g\right\|_{2} .
$$

Iterating this inequality $n$ times with $\kappa=t / n$,

$$
\sum_{\mathbf{I} \neq 0} w(\mathbf{I})\left\|P_{\mathbf{l}}(I-(t / n) \bar{\Omega})^{-n} g\right\|_{2} \leqq\left(1+(t / n) \lambda_{1}(1-\alpha)\right)^{-n}\|g\|_{a} .
$$

Let $n \rightarrow \infty$. We obtain from the Hille-Yosida Theorem:

$$
\sum_{\mathbf{l} \neq 0} w(\mathbf{l})\left\|P_{\mathbf{1}} S(t) g\right\|_{2} \leqq \exp \left\{-t \lambda_{1}(1-\alpha)\right\}\|g\|_{a} .
$$

To complete the proof of the theorem note that from (13) we obtain

$$
\left\|S(t) g-P_{\mathbf{o}} S(t) g\right\|_{u} \leqq \exp \left\{-t \lambda_{1}(1-\alpha)\right\}\|g\|_{a}
$$

so that if $\mu$ is an invariant measure for $\{S(t): t \geqq 0\}$,

$$
\left|\int g d \mu-P_{\mathbf{o}} S(t) g\right| \leqq \exp \left\{-t \lambda_{1}(1-\alpha)\right\}\|g\|_{a} .
$$

Equation (4) now follows from (14) and (15), completing the proof of the theorem.

Remark. The connection with Faris's uniqueness theorem is the following. We prove Lemma 1 with $B$ in the place of $B_{n}$, and conclude that

$$
\left\|B \circ A^{-1}\right\|<1 .
$$

Hence by Proposition $1, B \circ A^{-1}$ has operator norm $<1$ as an operator on $A(\Xi)$, and dually $A^{-1 * \circ B^{*}}$ has operator norm $<1$ on $A(\Xi)^{*}$, where a ${ }^{*}$ on an operator 
denotes Banach space adjoint. Thus the uniqueness and analyticity in $T^{-1}$ of the invariant measure follows from Faris's Proposition 1. Note that Faris uses the norm on measures dual to our norm on functions in $A(\Xi)$.

\section{Convergence to Equilibrium of the Plane-Rotor and Classical Heisenberg Models}

In this section we compute, for the plane-rotor and classical Heisenberg models, the temperature ranges over which Theorem 1 yields convergence to equilibrium. We carry out the computations simultaneously for both models.

For these models $M$ is either the circle $S^{1}$ (plane-rotor model) or the twosphere $S^{2}$ (the classical Heisenberg model), $\Delta$ is the usual Laplacian in the angle variables, and $\sigma$ is (normalized) surface measure. On the circle, each eigenspace $H_{l}$ has dimension two, with basis $\exp ( \pm i l \theta)$; on the two-sphere, $H_{l}$ has dimension $2 l+1$, with basis the spherical harmonics $\left\{Y_{l, m}:-l \leqq m \leqq l\right\}$ of degree 1 (normalized in $\left.L^{2}(M, \sigma)\right)$. $H_{l}$ has eigenvalue $\left(-l^{2}\right)$ on the circle and $(-l(l+1))$ on the sphere. $S$ is the lattice $Z^{v}$ of points in $R^{v}$ with integer coordinates.

The Hamiltonian is given (formally) in both models by:

$$
H=-\frac{1}{2} \sum_{\substack{x \\|x-y|=1}} \cos \Theta_{x y}
$$

where $\Theta_{x y}$ is the angle between $\xi(x)$ and $\xi(y)$. Thus

$$
B_{x}=\left(\nabla_{x} H\right) \cdot d_{x}=\sum_{\substack{y: \\|y-x|=1}} \sin \Theta_{x y} \partial / \partial \Theta_{x y} \cdot
$$

For the plane-rotor model with $\Theta_{x y}=\theta(x)-\theta(y)$ this may be written more simply as

$$
B_{x}=\sum_{\substack{y: \\|y-x|=1}} \sin (\theta(x)-\theta(y)) \partial / \partial \theta(x)
$$

The spaces $A(\Xi)$ are defined in both cases as in Sect.II. Note that the weight functions are

$$
w(\mathbf{l})=\prod_{x \in \operatorname{supp} \mathbf{l}} 2^{1 / 2}=2^{|\operatorname{supp}| / 2}
$$

for the plane-rotor model, and

$$
w(\mathbf{l})=\prod_{x \in \mathbf{s u p p} \mathbf{l}}(2 l(x)+1)^{1 / 2}
$$

for the Heisenberg model.

The computation of temperature ranges will follow from the two lemmas below. We give the proofs for the plane-rotor model. For the classical Heisenberg model they are found in [2].

Lemma 3. The operator $\sin \Theta_{x y} \partial / \partial \Theta_{x y}$ restricted to $H_{1}$ has norm bounded by $(-\lambda(l(x)))^{1 / 2}$. 
Proof. $H_{1}$ has an orthonormal basis consisting of the functions

$$
e_{1, \mathbf{m}}=\prod_{x} \exp \{i l(x) m(x) \theta(x)\}
$$

with $m(x)= \pm 1$ for all $x$. Thus if $Y_{1} \in H_{1},\left\|\sin (\theta(x)-\theta(y)) \partial / \partial \theta(x) Y_{1}\right\|_{2} \leqq l(x)\left\|Y_{1}\right\|_{2}$ $=\left(-\lambda(l(x))^{\frac{1}{1 / 2}}\left\|Y_{1}\right\|_{2}\right.$, since each of the basis functions (6) is an eigenfunction of $\partial / \partial \theta(x)$ with eigenvalue $\pm l(x)$ and $Y_{1}$ is a linear combination of these functions.

Lemma 4. Let $Y_{1} \in H_{1}$. If $y$ is a neighbor of $x$, then $\sin \Theta_{x y} \partial / \partial \Theta_{x y} Y_{1}$ is a sum of four terms belonging to spaces $H_{\mathbf{I}^{\prime}}$ with $l^{\prime}(x)=l(x) \pm 1, l^{\prime}(y)=l(y) \pm 1$ and $l^{\prime}(z)=l(z)$ for all other $z$.

Proof. Write $\sin (\theta(x)-\theta(y))$ as a linear combination of the functions $\exp \{ \pm i \theta(x)\}$. $\exp \{\mp i \theta(y)\}$.

We now compute a bound on the norm $\left\|B_{x}{ }^{\circ} A_{x}^{-1}\right\|$ of Theorem 1. Since $H_{1}$ is an eigenspace of $A_{x}$ of eigenvalue $\lambda(l(x))$ we need only find a bound on

$$
w(\mathbf{l})^{-1}(-\lambda(l(x)))^{-1} \sum_{\mathbf{l}^{\prime}} w\left(\mathbf{l}^{\prime}\right)\left\|P_{\mathbf{l}^{\prime}} B_{x} P_{\mathbf{1}}\right\|_{o p}
$$

for all $l$ with $l(x) \neq 0$. From the fact that $x$ has $2 v$ nearest neighbors and Lemma 4 we see that, if $Y_{1} \in H_{1}, B_{x} Y_{1}$ is a sum of $8 v$ terms belonging to various $H_{1^{\prime}}$. By the orthogonality of these spaces and Lemma 3, each of these terms is bounded by $\left(-\lambda(l(x))^{1 / 2} .\left\|Y_{1}\right\|_{2}\right.$. Thus (7) is less than

$$
8 v T^{-1}(-\lambda(l(x)))^{-1 / 2} \sup _{\mathbf{l}^{\prime}} w\left(\mathbf{l}^{\prime}\right) / w(\mathbf{l})
$$

where the supremum is over the various index functions described in Lemma 4 .

For the plane-rotor model $\left|\operatorname{supp} \mathbf{I}^{\prime}\right| \leqq|\operatorname{supp} \mathbf{I}|+1$ so that from $(4) w\left(\mathbf{l}^{\prime}\right) \leqq \sqrt{2} w(\mathbf{l})$ and so we have proved:

Theorem 2. For the plane-rotor model in $v$ dimensions we have the bound

$$
\left\|B_{x} \circ A_{x}^{-1} \mid\right\| \leqq \sqrt{2} 8 v T^{-1} .
$$

Thus if $T>\sqrt{ } 28 v$, the stochastic process converges to the equilibrium state.

For the classical Heisenberg model we have $2 l^{\prime}(x)+1 \leqq 2 l(x)+3,2 l^{\prime}(y)+1 \leqq$ $2 l(y)+3$ so that from $(5)$ we find $w\left(\mathbf{l}^{\prime}\right) / w(\mathbf{l}) \leqq \sqrt{5}$. Since $\lambda(l(x)) \geqq 2$ if $l(x) \neq 0$ we have proved:

Theorem 3. For the classical Heisenberg model in $v$ dimensions we have

$$
\left\|B_{x} \circ A_{x}^{-1} \mid\right\| \leqq \sqrt{5 / 2} 8 v T^{-1} .
$$

Thus the process converges to equilibrium if $T>\sqrt{5 / 2} 8 v$.

Remarks. The temperature range in Theorem 2 is considerably restricted vis à vis Faris's range $T>2 v$ over which he proved uniqueness and analyticity hold. In fact convergence to equilibrium occurs also over this range (see the Appendix). The range computed in Theorem 3 should be compared with Faris's result $T>8 v$. 


\section{Gibbs States, Reversible States and the Global Markov Property}

In the stochastic Heisenberg models, the vector field $X$ generating the drift part of the process is (formally) the gradient of a scalar function $H$-the Hamiltonian. The equation for an invariant (equilibrium) state is

$$
0=\int d \mu \Omega f
$$

for all smooth functions $f$. If we write $\mu=\rho \sigma$, as though $\mu$ had a density $\rho$ with respect to $\sigma$, this equation may be rewritten as

$$
0=\Omega^{t} \rho=\Delta \rho+T^{-1} \nabla \cdot(\rho \nabla H)
$$

where $\Omega^{t}$ is the formal adjoint (with respect to $\sigma$ ) of $\Omega$. As Faris observed in [1] the (formal) solution of this PDE is

$$
\rho=Z^{-1} \exp \left(-T^{-1} H\right)
$$

i.e. $\mu$ is the Gibbs state of the Hamiltonian.

If the set of sites $S$ is finite (so that $\Xi$ is a finite-dimensional manifold), this argument is valid and in fact $\mu$ is the unique equilibrium state of the process (see Prop. 2 below). When $S$ is infinite we must replace (1) by the rigorous definition of a Gibbs state formulated by Dobrushin, Lanford and Ruelle ([11], [12]). We shall then identify a class of equilibrium states (called reversible states) and prove that this class is identical with the class of Gibbs states. (For the stochastic Ising model this was proved in [13]; see also [5].) Thus Theorem 1 (implies convergence, at sufficiently high temperature, of the stochastic process with arbitrary initial state to the (unique) Gibbs state of the Hamiltonian. On the other hand it was shown in [14] that in the classical Heisenberg model with $v \geqq 3$ at low temperatures the Gibbs state is not unique. The implication is that there exist distinct equilibrium states of the stochastic process, at low temperatures.

We also discuss in this section an additional property of the equilibrium state, valid when Faris's uniqueness theorem holds: the global Markov property.

We begin with the rigorous definition of a Gibbs state. We assume that $H$ is given formally by:

$$
H=-\sum_{p \subset S} J(F)
$$

where the sum is over finite subsets of $S$ and the $J(F)$ are smooth functions of $\xi(x), x \in F$ called potentials. (For example, in the classical Heisenberg model $J(\{x, y\})=\xi(x) \cdot \xi(y)$ if $x$ and $y$ are neighbors, and $J(F)=0$ for all other $F$.) In order to satisfy the finite-range condition we assume $\exists r<\infty$ such that $J(F)=0$ if $\operatorname{diam} F>r$. Now let $\Lambda \subset S$ be a finite set and $\zeta \in M^{S \backslash \Lambda}$ an external configuration. We define the conditional Gibbs state inside $\Lambda$ with boundary condition $\zeta$ by:

$$
d \mu_{\Lambda, \zeta}=Z_{\Lambda, \zeta}^{-1} \exp \left(-T^{-1} H_{\Lambda, \zeta}\right) d \sigma_{\Lambda},
$$

where $\sigma_{\Lambda}$ is the product of the normalized volume measures on $M_{x}$ for $x \in \Lambda$, $Z_{\Lambda, \zeta}$ is a normalization factor, and $H_{\Lambda, \zeta}$ is the energy of the configuration inside 
$\Lambda$, given the boundary condition $\zeta$. The latter is defined by

$$
H_{\Lambda, \zeta}(\xi)=-\sum_{F \cap \Lambda \neq \phi} J(F)(\xi \cdot \zeta)
$$

where $\xi \cdot \zeta$ is the configuration equal to $\xi$ on $\Lambda$ and $\zeta$ on $S \backslash \Lambda$ (note however that $H_{\Lambda, \zeta}$ depends only on $\zeta(y)$ for dist $\left.(y, \Lambda) \leqq r\right)$.

The Gibbs states are now defined as follows. A state $\mu$ on $\Xi$ is a Gibbs state of the Hamiltonian (at temperature $T$ ) iff $\mu$ admits the function $\mu_{\Lambda, \zeta}$ as a regular conditional probability, given the configuration $\zeta$ in $S \backslash \Lambda$. Thus $\mu$ is a Gibbs state iff for all (conditions) functions $f, g$ with $g$ depending only on the configuration $\zeta$ outside $\Lambda$ we have:

$$
\int d \mu g f=\int d \mu(\zeta) g(\zeta) \int d \mu_{\Lambda, \zeta}(\xi) f(\xi \cdot \zeta) .
$$

(These equations are usually called DLR equations.)

Returning to the stochastic process we will call a state $\mu$ on $\Xi$ reversible for the semigroup $\{S(t): t \geqq 0\}$ if for all $f, g$ in $C(\Xi)$ and $t \geqq 0$,

$$
\int d \mu f S(t) g=\int d \mu g S(t) f
$$

An equivalent definition is: For all $f, g$ in (a core of) the domain of the generator $\Omega$,

$$
\int d \mu f \Omega g=\int d \mu g \Omega f
$$

Clearly a reversible state is invariant (take $f \equiv 1$ in (6)).

We are now ready to state our theorem.

Theorem 4. Let $H$ be defined formally by (2) with potentials satisfying the finiterange condition. Assume that there exists a Markov semigroup on $C(\Xi)$ with generator the closure of $\Omega=\Delta-T^{-1} \nabla H \cdot d$ (with the usual domain). Then the class of reversible states for this semigroup coincides with the class of Gibbs states of $H$.

Proof. We require the following proposition, proved by Nelson in [15].

Proposition 2. Let $N$ be a compact, finite-dimensional Reimannian manifold and $X$ a smooth vector field on $N$. Then the generator

$$
\Omega=\Delta+X \cdot d
$$

has a unique invariant probability measure $\mu$, which is reversible iff $X$ is the gradient of a scalar function $\Psi$. In this case $\mu$ is given by

$$
d \mu=Z^{-1} \exp (\Psi) d \sigma,
$$

where $\sigma$ is the volume measure on $N$.

Let $\mu$ be a Gibbs state of $H$. Let $\Lambda \subset S$ be a finite set. For any external configuration $\zeta$ define a generator $\Omega_{\Lambda, \zeta}$ on $N=M^{\Lambda}$ by taking $X=-T^{-1} \nabla H_{\Lambda, \zeta}$. Then by the proposition, $\mu_{\Lambda, \zeta}$ is the unique reversible measure of $\Omega_{\Lambda, \zeta}$ so that if $f, g$ are smooth functions on $M^{\Lambda}$,

$$
\int d \mu_{\Lambda, \zeta} f \Omega_{\Lambda, \zeta} g=\int d \mu_{\Lambda, \zeta} g \Omega_{\Lambda, \zeta} f .
$$

We then obtain from (5) by integrating both sides of (11) with $\mu$ (note that if $f$ is a 
function on $M^{\Lambda}$ then $\Omega_{\Lambda, \zeta} f(\xi)=\Omega f(\xi \cdot \zeta)$ if we regard $f$ as a function on $\Xi$ ):

$$
\int d \mu f \Omega g=\int d \mu g \Omega f .
$$

Thus $\mu$ is reversible.

Conversely assume that $\mu$ is reversible for the process with generator $\Omega$. First note that if $f \in C^{2}\left(M^{\Lambda}\right), g \in C^{2}\left(M^{\Lambda^{\prime}}\right)$ with $\Lambda \cap \Lambda^{\prime}=\phi$, then

$$
\Omega(f g)=f \Omega g+g \Omega f .
$$

Since $\mu$ is reversible, it is invariant and so we obtain from (7) and (12)

$$
0=\int d \mu \Omega(f g)=2 \int d \mu g \Omega f .
$$

We may rewrite this as

$$
0=\int d \mu(\zeta) g(\zeta) \int d \mu(\xi \mid S \backslash \Lambda)(\zeta) \Omega_{\Lambda, \zeta} f(\xi),
$$

where $\zeta \rightarrow \mu(\cdot \mid S \backslash \Lambda)(\zeta)$ is a regular conditional probability for $\mu$, given the configuration $\zeta$ in $S \backslash \Lambda$. Letting $f$ and $g$ range over countable dense sets in $C^{2}\left(M^{\Lambda}\right)$ and $C\left(M^{S \backslash A}\right)$ respectively we obtain

$$
0=\int d \mu(\xi \mid S \backslash \Lambda)(\zeta) \Omega_{\Lambda, \zeta} f(\xi), \mu-\text { a.e. }
$$

Thus for $\mu$-a.e. $\zeta, \mu(\cdot \mid S \backslash \Lambda)(\zeta)$ is an invariant state for $\Omega_{\Lambda, \zeta}$. From the proposition

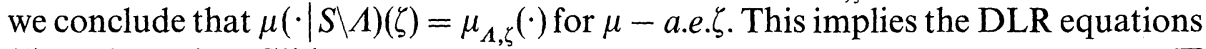
(5) so that $\mu$ is a Gibbs state.

A probability measure $\mu$ on $\Xi=M^{S}$ is said to have the local Markov property (LMP) of range $r$ if for all finite $\Lambda \subset S$,

$$
\mu(\cdot \mid S \backslash \Lambda)(\zeta)=\mu\left(\cdot \mid \partial_{r} \Lambda\right)(\zeta), \mu-\text { a.e. }
$$

where $\mu(\cdot \mid S \backslash \Lambda)(\zeta)$ (respectively $\left.\mu\left(\cdot \mid \partial_{r} \Lambda\right)(\zeta)\right)$ is the conditional probability given the configuration in $S \backslash \Lambda$ (respectively in $\partial_{r} \Lambda=\{y$ : dist $(y, \Lambda) \leqq r\}$ ). If $\mu$ is a Gibbs state of a Hamiltonian with potentials of range $\leqq r$ then $\mu$ has the LMP of range $r$.

The global Markov property (GMP) of range $r$ is said to hold if we may take $A$ arbitrary in (4). The GMP is not an immediate consequence of the LMP; in fact there exists a Gibbs state of the Ising model for $v=3$ which fails to have the GMP [16]. The GMP follows from Dobrushin's uniqueness theorem for the Gibbs state [17], and holds also for the extreme states in FKG ordering of the Ising model [16], [18].

Theorem 5. The unique equilibrium state (Gibbs state) of the plane-rotor and classical Heisenberg models has the GMP whenever Faris's uniqueness theorem (or Theorem 1) applies.

Proof. Let $\mu$ be the unique invariant state and $\Lambda \subset S$ an arbitrary set. Given a configuration $\zeta$ in $\partial_{r} \Lambda$, define $H_{\Lambda, \zeta}$ formally by (4) and let $\Omega_{\Lambda, \zeta}=\Delta_{\Lambda}-T^{-1} \nabla H_{\Lambda, \zeta} \cdot d$ $\left(\Delta_{\Lambda}=\sum_{x \in \Lambda} \Delta_{x}\right)$. Since $\mu$ is reversible, (13) holds for $\mu$-a.e. $\zeta$. An examination of the bounds on $\left\|P_{1}, B_{x} P_{1}\right\|_{o p}$ proved in Sect. IV and in [2] reveals that these same bounds are valid with $B_{\Lambda, \zeta, x}=T^{-1} \nabla_{x} H_{\Lambda, \zeta} \cdot d_{x}$ replacing $\mathrm{B}_{x}$, provided $x \in \Lambda$ and the support of $\mathbf{l}$ and $\mathbf{l}^{\prime}$ lie in $\Lambda$. (The proofs of these bounds required only the deter- 
mination of the spaces containing the various terms and $a$ bound on these terms as functions of $\xi(x)$ which is uniform in $\xi(y)$ for $y \neq x$.) Thus we may invoke Faris's. (uniqueness) theorem or Theorem 1 for the process generated by (the closure of) $\Omega_{\Lambda, \zeta}$ and conclude that it has a unique invariant measure $\mu_{\Lambda, \zeta}$. Furthermore $\mu_{\Lambda, \zeta}$ is an (analytic) function of $B_{\Lambda, \zeta}$, which in turn depends on $\zeta(y)$ only for $y \in \partial_{r} \Lambda$. Thus $\mu_{\Lambda, \zeta}(\cdot)$ is $\partial_{r} \Lambda$ measurable in $\zeta$. From (13) we conclude that $\mu(\cdot \mid S \backslash \Lambda)(\zeta)=\mu_{\Lambda, \zeta}(\cdot)$, $\mu$-a.e. Thus $\mu(\cdot \mid S \backslash \Lambda)(\zeta)$ is $\partial_{r} \Lambda$-measurable and (14) holds a fortiori.

\section{Appendix. A Sharpened Version of Theorem 1 for the Plane-Rotor Model}

The restricted temperature range computed in Theorem 2 for the plane-rotor model stems from a somewhat unnatural choice in this case of the space $A(\Xi)$. In fact if $M=S^{1}$ (unit circle), $\Xi=\left(M^{S}\right)$ is a commutative group, and the functions defined in Eq. (A6) of Sect. IV are characters of $\Xi$. It is not hard to show that convergence of the Laplace series $\sum_{\mathbf{l}}\left(\sum_{\mathbf{m}} a_{\mathbf{l}, \mathbf{m}} e_{\mathbf{l}, \mathbf{m}}\right)$ in $A(\Xi)$ implies $\sum_{\mathbf{l}, \mathbf{m}}\left|a_{\mathbf{l}, \mathbf{m}}\right|<\infty$, i.e. absolute convergence of the Fourier series in the usual sense. This suggests that to obtain a strengthened version of Theorem 2 we should use more conventional methods (and notation) of Fourier analysis.

Let $\Xi=\left(S^{1}\right)^{S}$ and let $\hat{\Xi}$ be the character group of $\Xi$. $\hat{\Xi}$ is isomorphic to the additive group of functions $\mathbf{n}: S \rightarrow Z$ with only finitely many non-zero values, via the map $\mathbf{n} \rightarrow \gamma_{\mathbf{n}}$, where $\gamma_{\mathbf{n}}$ is the character

$$
\gamma_{\mathbf{n}}=\exp \left(i \sum_{x} n(x) \theta(x)\right)
$$

In this context $\sigma$ is the normalized Haar measure on $\Xi$. Define the Fourier coefficients of a function $f \in L^{2}(\Xi, \sigma)$ by:

$$
\hat{f}(\mathbf{n})=\int d \sigma f \bar{\gamma}_{\mathbf{n}}
$$

The series

$$
\sum \hat{f}(\mathbf{n}) \gamma_{\mathbf{n}}
$$

then converges in $L^{2}(\Xi, \sigma)$ to $f$. Let $A^{\sim}(\Xi)=\left\{f \in L^{2}(\Xi, \sigma): \sum_{\mathbf{n}}|f(\mathbf{n})|<\infty\right\}$ with norm

$$
\|f\|_{a}=\|\hat{f}\|_{1}=\Sigma|\hat{f}(\mathbf{n})| \text {. }
$$

Finally if $\Gamma$ is a bounded operator on $L^{2}(\Xi, \sigma)$ let

$$
\|\Gamma\|^{\sim}=\sup _{\mathbf{n}} \sum_{\mathbf{n}^{\prime}}\left|\Gamma_{\mathbf{n}^{\prime}, \mathbf{n}}\right|,
$$

where $\Gamma_{\mathbf{n}^{\prime}, \mathbf{n}}=\left(\gamma_{\mathbf{n}^{\prime}}, \Gamma \gamma_{\mathbf{n}}\right)_{2}$ are the matrix elements of $\Gamma$. In this case $\|\Gamma \mid\| \sim$ is, if finite, the operator norm of $\Gamma$ as an operator on $A^{\sim}(\Xi)$.

We are now able to state a theorem which is considerably easier to use in this setting than Theorem 1 . We continue the notational conventions adopted in the first paragraph of Sect. III. $X$ is a vector field with smooth coefficients satisfying the finite-range condition and $\Omega=A+B=\Delta+T^{-1} X \cdot d$. 
Theorem 6. Let $\Xi$ be the commutative group $\left(S^{1}\right)^{S}$. Assume $\exists \alpha<1$ such that

$$
\left\|B_{x}^{\circ} A_{x}^{-1}\right\| \|^{\sim} \leqq \alpha
$$

for all $x \in S$. Then the conclusions of Theorem 1 (stated with the norm $\|\cdot\|_{a}$ ) follow for the generator $\bar{\Omega}$.

Proof. Let $H_{\mathbf{n}}$ be the one-dimensional space spanned by $\gamma_{\mathbf{n}}$ so that $w(\mathbf{n})=1$ for all $\mathbf{n}$. Now the proof of Theorem 1 applies verbatim.

By exploiting the group structure of the characters we may state a corollary which applies to more general Hamiltonians than the one defined by Eq. (1) of Sect. IV. It is convenient to assume that $H$ has the formal Fourier series

$$
H=\sum_{\mathbf{n}} \hat{H}(\mathbf{n}) \gamma_{\mathbf{n}}
$$

If we impose the condition

$$
\sum_{\mathbf{n}}|\hat{H}(\mathbf{n})||n(x)|<\infty
$$

for all $x \in S$, then the operator $B_{x}=-T^{-1} \nabla_{x} H \cdot d_{x}$ is well-defined and equals

$$
-T^{-1} i\left\{\sum_{\mathbf{n}} \hat{H}(\mathbf{n}) n(x) \gamma_{\mathbf{n}}\right\} \partial / \partial \theta(x)
$$

We also impose the restriction

$$
\hat{H}(\mathbf{n})=0 \text { if diam supp } \mathbf{n}>r,
$$

which implies the finite-range condition.

Corollary. Let $H$ be defined formally by (A3) with Fourier coefficients satisfying (A6). Assume that $\exists \alpha<1$ such that

$$
\sum_{\mathbf{n}}|\hat{H}(\mathbf{n})||n(x)| \leqq \alpha T
$$

for all $x \in S$. Then the closure of $\Omega=\Delta-T^{-1} \nabla H \cdot d$ generates a Markov semigroup converging to equilibrium (as defined in Sect. I.)

Proof. A quick calculation gives the matrix element $\Gamma_{\mathbf{n}^{\prime}, \mathbf{n}}(x)$ of $B_{x} \circ A_{x}^{-1}$ :

$$
\Gamma_{\mathbf{n}^{\prime} \mathbf{n}}(x)=T^{-1} n(x)^{-1} \hat{H}\left(\mathbf{n}^{\prime}-\mathbf{n}\right)\left(n^{\prime}(x)-n(x)\right)
$$

if $n(x) \neq 0$. The bound (2) of Theorem 6 then follows from (A1) and (A7).

Returning to the Hamiltonian of the usual plane-rotor model (Eq. (1) of Sect.IV) we easily compute the Fourier coefficients: $\hat{H}(\mathbf{n})=1 / 2$ if $\operatorname{supp} \mathbf{n}=\{x, y\}$ with $n(x)=1, n(y)=-1 ; \hat{H}(\mathbf{n})=0$ for all other $\mathbf{n}$. Thus

$$
\sum_{\mathbf{n}}|H(\mathbf{n})||n(x)| \leqq 4 v \cdot 1 / 2=2 v
$$

and so we have proved

Theorem 7. The stochastic plane-rotor model in $v$ dimensions converges to equilibrium if $T>2 v$. 
Acknowledgements. The author would like to thank D. Ragozin, S. Albeverio, and W. Faris for valuable discussions, and especially his thesis advisor, J. Westwater, for his advice and encouragement.

\section{References}

1. Faris, W. H. : J. Funct. Anal. 32, 342-352 (1979)

2. Faris, W. H.: Trans. Am. Math. Soc. 261. 579-587 (1980)

3. Liggett, T. M.: The stochastic evolution of infinite systems of interacting particles. In : Ecole d'Eté de Saint-Flour VI-1976. Lecture Notes in Mathematics, Vol. 598, Springer, Berlin, Heidelberg, New York 1977

4. Ito, K. : Stochastic processes 1968/69. Aarhus, Aarhus Universitet, Matematisk Institut (1969)

5. Holley, R. Stroock, D. : Z. Wahrsch. Verw. Gebiete 35, 87-101 (1976)

6. Holley, R. Stroock, D. : Ann. Probab. 4, 195-228 (1976)

7. Ragozin, D. L. : Math. Ann. 219, 1-11 (1976)

8. Peetre, J. : Math. Ann. 169, 307-314 (1967)

9. Stein, E. M. Weiss, G. : Introduction to Fourier analysis on euclidean spaces. Princeton, N. J. : Princeton University Press, 1971

10. Yosida, K. : Nagoya Math. J. 3, 1-4 (1951)

11. Dobrushin, R. L. : Theory Probab. Its Appl. 13, 197-224 (1968)

12. Lanford, O. E. Ruelle, D. : Commun. Math. Phys. 13, 194-215 (1969)

13. Dobrushin, R. L. : Probl. Peredachi Inf. 7, 57-66 (1971)

14. Fröhlich, J., Simon, B., Spencer, T. : Commun. Math. Phys. 50, 79-85 (1976)

15. Nelson, E. : Duke Math. J. 25, 671-690 (1958)

16. Goldstein, S. : Commun. Math. Phys. 74, 223-234 (1980)

17. Albeverio, S., Hoegh-Krohn, R., Olsen, G. : The global Markov property for lattice systems. Preprint, Bielefeld-Marseille (1978)

18. Folmer, H. : On the global Markov property. Preprint (1978)

Communicated by J. L. Lebowitz

Received January 23, 1981 
